\title{
Caracterização da demanda e procura de um pronto atendimento infantil: opinião de pais e responsáveis
}

\section{Demand characterization and looking for a children's emergency care: opinion of parents and legal guardians.}

\author{
Bruna Antoniassi Silva ${ }^{1}$; Amanda Mello de Oliveira²; Edilaine Giovanini Rossetto \\ Adriana Valongo Zani ${ }^{4}$
}

\begin{abstract}
Resumo
Os serviços de saúde são organizados de forma hierárquica a fim de se obter melhor resolução e universalização do acesso. No entanto, na prática, isto não tem ocorrido devido à limitação de serviços na atenção primária, e a porta de entrada tem sido os hospitais, através dos prontos atendimentos, prontos socorros ou ambulatórios, pois muitos acreditam ser a forma de atendimento mais rápida e eficaz. Portanto, o objetivo deste estudo foi identificar as causas que levam pais/responsáveis a escolher um pronto atendimento infantil para a assistência de seus filhos. Trata-se de um estudo descritivo e transversal de abordagem quantitativa. A amostra foi constituída por 380 pais/responsáveis, o local de coleta foi um pronto atendimento infantil do município de Londrina, no período de junho a agosto de 2013. Após análise dos dados, observou-se que 73,9\% da população recorreram ao pronto atendimento infantil mediante a procura direta, a pequena parcela que procurou por outro serviço anteriormente apenas $12,6 \%$ foi encaminhado. A queixa principal foi febre com $25,7 \%$ e casos de convulsão apareceram em último lugar com $0,4 \%$. O presente estudo revelou uma grande procura da população estudada pelos serviços de prontos atendimentos em detrimento a atenção primária, com queixas, muitas vezes, passíveis de resolução na atenção primária, o que confirma a deficiência em alguns princípios prioritários pelo Sistema Único de Saúde, como acessibilidade e resolutividade dos serviços de saúde primária.
\end{abstract}

Palavras-chave: Estresse. Centro cirúrgico. Equipe de enfermagem.

\begin{abstract}
Health services are organized hierarchically in order to obtain better resolution and universal access. However, in practice, this has not occurred due to the limitation of services in primary care, and the gateway has been hospitals, through the ready attendance, emergency rooms or clinics because many believe it is the fastest and most effective form of care. Therefore, the objective of this study was to identify the reasons for parents/guardians to choose the ready attendance service for the assistance of their children. It is a descriptive and cross-sectional study with a quantitative approach. The sample consisted of 380 parents/guardians, the collection site was a children's health care center in Londrina, from June to August 2013. After analyzing the data, it was observed that $73.9 \%$ of the population resorted directly to the ready attendance service. From
\end{abstract}

\footnotetext{
${ }^{1}$ Enfermeira. Residente em Enfermagem neonatal pela Universidade Estadual de Londrina.

${ }^{2}$ Graduanda do Curso de Enfermagem da Universidade Estadual de Londrina.

${ }^{3}$ Enfermeira. Doutora. Co-orientadora. Docente do Departamento de Enfermagem e Residência de Enfermagem Neonatal da Universidade Estadual de Londrina.

${ }^{4}$ Enfermeira. Doutora. Orientadora. Docente do Departamento de Enfermagem e Residência de Enfermagem Neonatal da Universidade Estadual de Londrina.
} 
the small portion that searched for other hospitals, only $12.6 \%$ were sent to the ready attendance service. The main complaint was fever with $25.7 \%$ and cases of seizures appeared in last place with $0.4 \%$. The present study revealed a great demand of the population studied by ready attendance services instead of primary care, with complaints often solvable in primary care, which confirms the deficiency in priority principles by the Unified Health System such as accessibility and response capacity of primary health services.

Keywords: Children's emergency care. Child care. Primary healthcare.

\section{Introdução}

As metas de descentralização e hierarquização no atendimento pediátrico não têm conseguido obter o êxito esperado, pois altas demandas por atendimentos nos serviços de urgência ainda persistem (PEIXOTO et al., 2013).

Essas altas demandas dos serviços evidenciam as dificuldades do sistema em atingir metas de universalidade, igualdade e descentralização do acesso à atenção à saúde. A fragilidade dos sistemas de referência e contrarreferência é um fator determinante do volume de atendimentos. Poucos preferem a unidade básica quando a criança adoece, e a maioria utiliza os serviços de urgência, sendo justificado pelo gosto/confiança nos profissionais, acessibilidade geográfica, qualidade e rapidez do atendimento, experiência prévia pessoal e resolutividade (PEIXOTO et al., 2013; SILVA; TRONCHIN, 2011).

A Atenção Básica tem sido definida no Brasil como sinônimo de atenção primária, ou seja, é o primeiro nível de assistência no sistema de saúde e tem elementos estruturais e funcionais, caracterizando-se, principalmente, pela universalidade, integralidade, equidade, acessibilidade, humanização, responsabilidade e coordenação da assistência (BRASIL, 2007; STARFIELD, 2002).

A criança é considerada como um todo em suas múltiplas relações, e a família é valorizada, assim como o contexto em que a criança vive. Os princípios como acesso aos serviços de saúde e assistência integral são destacados com diferentes níveis de atenção. Facilitar o acesso e promover a saúde da criança, incluindo o desenvolvimento de ações para prevenir danos e prestar assistência, quando há complicações, pode melhorar a assistência à saúde da criança e, consequentemente, a qualidade de vida da criança (FURTADO et al., 2013). Portanto, o Ministério da Saúde elaborou o protocolo de acolhimento por classificação de risco, não apenas para os serviços de emergências e urgências, mas também para atenção primária, pois todos estes princípios necessários à qualidade da assistência devem ocorrer, possibilitando que até nos casos em que seja necessária a transferência/encaminhamento da criança a outros pontos de atenção, este possa ocorrer de modo efetivo (BRASIL, 2007).

O acolhimento é uma proposta de reorganização dos serviços para garantir o acesso com resolutividade e atendimento humanizado, em uma tentativa de minimizar os obstáculos. Este propõe que o serviço de saúde seja organizado de forma usuário-centrada, partindo dos seguintes princípios: atender a todas as pessoas que procuram os serviços de saúde, garantindo a acessibilidade universal; reorganizar o processo de trabalho, a fim de que este desloque seu eixo central do médico para uma equipe multiprofissional equipe de acolhimento, encarregada de escutar o usuário e comprometendo-se a resolver seu problema de saúde (SILVA; TRONCHIN, 2011).

Neste sentido, a Atenção Básica (AB) deve efetuar algumas funções para contribuir com o funcionamento das Redes de Atenção à Saúde, como ser base para o serviço de saúde, ser resolutiva, coordenar o cuidado através da comunicação entre diversos pontos de atenção, ordenar as redes reconhecendo as necessidades de saúde dos usuários (BRASIL, 2013). 
No entanto, na prática, isso não ocorre devido à limitação de serviços na atenção primária, e a porta de entrada tem sido os hospitais, através dos prontos atendimentos (PA), prontos socorros (PS) ou ambulatórios, pois muitos acreditam ser a forma de atendimento mais rápida e eficaz (MARQUES; LIMA, 2007). Com isso o ambiente do pronto atendimento (PA) é marcado pela aglomeração de pacientes, gerando pressão temporal com interferências diretas sobre o ato pediátrico praticado (SILVA; TRONCHIN, 2011).

Diante disso, o objetivo deste estudo foi identificar as causas que levam pais/responsáveis a escolher um PA para a assistência de seus filhos.

\section{Material e Método}

Trata-se de um estudo descritivo e transversal de abordagem quantitativa. Sendo desenvolvido em um Pronto Atendimento Infantil (PAI) no município de Londrina-PR, que tem a função assistencial de atender as necessidades de crianças portadoras de quadros agudos sendo esses de origem clínica ou traumática.

Os sujeitos que participaram da pesquisa foram pais e/ou responsáveis que levaram seus filhos para serem atendidos no PAI, independente do sexo ou idade e que aceitaram participar da pesquisa.

A amostra por conveniência foi calculada a partir do número infinito, constituindo 380 pais/responsáveis que estavam presentes no PAI aguardando seu filho (os) ser atendido e responderam às entrevistas, durante os meses de junho a agosto de 2013.

A pesquisa foi realizada mediante parecer favorável do Comitê de Ética em Pesquisa da Universidade Estadual de Londrina - UEL, sob $\mathrm{n}^{\circ} 301.276$ e autorização dos pais/responsáveis e atendeu os princípios da Resolução no466, de 12 de dezembro de 2012, a qual regulamenta pesquisas envolvendo seres humanos (BRASIL, 2012).

Para a coleta de dados foi utilizado um questionário com questões fechadas contendo duas partes. A primeira teve a finalidade de obter dados sociodemográficos para caracterização da amostra: idade, escolaridade e grau de parentesco dos pais ou responsável, bem como renda familiar, região de moradia e idade da criança. A segunda parte foi composta por questões relacionadas ao itinerário percorrido, motivo de procura e satisfação pelo atendimento.

Os dados coletados foram transcritos para uma planilha no programa Excel e, posteriormente, realizado análise descritiva, por meio do programa estatístico Statistical Package for the Social Sciences (SPSS).

\section{Resultados}

As variáveis foram agrupadas em três blocos, compondo três tabelas de dados: a primeira refere-se ao perfil sociodemográfico dos pais/ responsáveis e das crianças, a segunda refere-se à avaliação dos serviços procurados anteriores ao PAI e a terceira aborda as causas da procura do mesmo.

Em relação ao perfil sociodemográfico dos participantes do estudo $71,6 \%$ apresentavam faixa etária entre 20 a 34 anos, destes $85 \%$ eram mães, 78,7\% possuíam companheiro. Em relação à escolaridade 68,7\% apresentam mais de oito anos de estudo, a maioria possuía ocupação $(55,7 \%)$ e com renda familiar inferior a três salários mínimos $(95,0 \%)$. Um número significativo $(38,2 \%)$ reside na região norte do município. No que tange as crianças atendidas pelo PAI, 206 eram do sexo masculino $(54,2 \%)$ e 279 estavam na faixa etária de 0 a 3 anos $(73,4 \%)$ como demonstrado na Tabela 1. 
Tabela 1 - Perfil sociodemográfico dos pais/responsáveis e crianças atendidas no Pronto Atendimento Infantil no município de Londrina - PR, 2013.

\begin{tabular}{|c|c|c|}
\hline Variáveis & $\mathbf{n}$ & $\%$ \\
\hline \multicolumn{3}{|l|}{ Pais /responsáveis } \\
\hline \multicolumn{3}{|l|}{ Idade (anos) } \\
\hline 15 a 19 & 48 & 12,6 \\
\hline 20 a 34 & 272 & 71,6 \\
\hline 34 e mais & 60 & 15,8 \\
\hline \multicolumn{3}{|l|}{ Estado civil } \\
\hline Com companheiro & 299 & 78,7 \\
\hline Sem companheiro & 81 & 21,3 \\
\hline \multicolumn{3}{|c|}{ Escolaridade (em anos)* } \\
\hline Até 8 & 118 & 31,1 \\
\hline$>8$ & 261 & 68,7 \\
\hline \multicolumn{3}{|l|}{ Ocupação } \\
\hline Sem ocupação & 168 & 44,3 \\
\hline Com ocupação & 212 & 55,7 \\
\hline \multicolumn{3}{|l|}{ Grau de parentesco } \\
\hline Mãe & 325 & 85,5 \\
\hline Pai & 40 & 10,5 \\
\hline Outros & 15 & 3,9 \\
\hline \multicolumn{3}{|c|}{ Renda familiar (salários mínimos)* } \\
\hline 1 a 3 & 361 & 95,0 \\
\hline 4 e mais & 17 & 4,5 \\
\hline \multicolumn{3}{|l|}{ Região de Moradia } \\
\hline Norte & 145 & 38,2 \\
\hline Leste & 87 & 22,9 \\
\hline Sul & 60 & 15,8 \\
\hline Oeste & 53 & 13,9 \\
\hline Fora de Londrina & 18 & 4,7 \\
\hline Centro & 17 & 4,5 \\
\hline \multicolumn{3}{|l|}{ Criança } \\
\hline \multicolumn{3}{|l|}{ Idade } \\
\hline$<1$ ano & 114 & 30,0 \\
\hline 1 a 3 anos & 165 & 43,4 \\
\hline$>4$ anos & 101 & 26,6 \\
\hline \multicolumn{3}{|l|}{ Sexo } \\
\hline Feminino & 174 & 45,8 \\
\hline Masculino & 206 & 54,2 \\
\hline Total & 380 & 100 \\
\hline
\end{tabular}

* Excluído informações ignoradas

Fonte: Do autor. 
Observa-se na tabela 2 que em relação à avaliação dos pais/responsáveis sobre os atendimentos anteriores a procura pelo PAI, da amostra total apenas 99 procurou por serviços anteriores. Destes $86(22,7 \%)$ buscaram os serviços da Unidade Básica de Saúde (UBS). No entanto, apenas 68 foram avaliados, sendo, na sua maioria, por médicos (52,9\%). Em relação ao grau de satisfação com o atendimento recebido, mais da metade
$(58,6 \%)$ avaliou como satisfatório. Porém, pode-se observar que, do total de entrevistados $(\mathrm{n}=380)$, quase metade $(49,5 \%)$ está insatisfeito com o atendimento na UBS. No que tange as causas da satisfação com o atendimento, destaca-se a agilidade com $73,6 \%$, e em relação às causas de insatisfação $(82,5 \%)$, destacam-se demora no atendimento, dificuldade de agendamento de consulta e falta de médico/pediatra.

Tabela 2 - Avaliação dos serviços procurados anteriores ao serviço de pronto atendimento infantil no município de Londrina - PR, 2013.

\begin{tabular}{|c|c|c|}
\hline Variáveis & n & $\%$ \\
\hline \multicolumn{3}{|l|}{ Serviço procurado $(n=99)$} \\
\hline UBS & 86 & 22,7 \\
\hline Hospital secundário & 6 & 1,7 \\
\hline Hospital terciário & 7 & 1,8 \\
\hline \multicolumn{3}{|c|}{ Categoria profissional que realizou avaliação $(n=68)$} \\
\hline Enfermeiro & 31 & 45,6 \\
\hline Médico & 36 & 52,9 \\
\hline Outros & 1 & 1,5 \\
\hline \multicolumn{3}{|c|}{$\begin{array}{l}\text { Grau de satisfação em relação ao atendimento do profissio- } \\
\text { nal }(n=70)\end{array}$} \\
\hline Satisfatório & 41 & 58,6 \\
\hline Parcialmente satisfatório & 11 & 15,7 \\
\hline Insatisfatório & 15 & 21,4 \\
\hline Não foi atendido & 3 & 4,3 \\
\hline \multicolumn{3}{|c|}{$\begin{array}{l}\text { Avaliação dos pais sobre o atendimento na UBS } \\
(n=380)\end{array}$} \\
\hline Satisfatório & 182 & 47,9 \\
\hline Insatisfatório & 188 & 49,5 \\
\hline Não frequenta & 10 & 2,6 \\
\hline \multicolumn{3}{|c|}{ Causas da Satisfação com o atendimento na UBS } \\
\hline Agilidade no atendimento & 117 & 73,6 \\
\hline Profissionais competentes & 42 & 26,4 \\
\hline \multicolumn{3}{|c|}{ Causas da Insatisfação com o atendimento na UBS } \\
\hline Falta de médico/pediatra & 94 & 38,2 \\
\hline Demora no atendimento & 72 & 29,3 \\
\hline Dificuldade para agendamento de consulta & 37 & 15,0 \\
\hline Mau atendimento & 24 & 9,7 \\
\hline Falta de medicamentos & 14 & 5,7 \\
\hline Falta de organização & 5 & 2,1 \\
\hline
\end{tabular}

Fonte: Do autor. 
Quanto à caracterização da procura pelo serviço de PAI (tabela 3 ) observa-se que a febre $(25,7 \%)$, tosse $(22,2 \%)$ e dor $(11,7 \%)$ foram as principais causas de procura por atendimento. Sendo o período da tarde escolhida por mais da metade $(55,8 \%)$. Os principais motivos pela escolha de período para atendimento foram a indisponibilidade nos demais períodos $(19,7 \%)$, por acreditar ser o período mais rápido $(16,8 \%)$ e possuir menor número de pessoas para atendimento (16,3\%). Da amostra total, a maioria $(87,6 \%)$ já havia sido atendida anteriormente no PAI. Destes $87,4 \%$ não possuíam encaminhamento.

Tabela 3 - Caracterização da procura pelo serviço de pronto atendimento infantil no município de Londrina - PR, 2013.

\begin{tabular}{|c|c|c|}
\hline Variáveis & n & $\%$ \\
\hline \multicolumn{3}{|l|}{ Causas da procura $(n=684)$} \\
\hline Febre & 176 & 25,7 \\
\hline Tosse & 152 & 22,2 \\
\hline Dor & 80 & 11,7 \\
\hline Outras queixas & 77 & 11,2 \\
\hline Dificuldade respiratória & 68 & 9,9 \\
\hline Vomito & 64 & 9,5 \\
\hline Diarreia & 20 & 2,9 \\
\hline Acidente & 20 & 2,9 \\
\hline Dor de ouvido & 19 & 2,9 \\
\hline Sangue nas fezes & 5 & 0,7 \\
\hline Convulsão & 3 & 0,4 \\
\hline \multicolumn{3}{|l|}{ Periodo de procura $(n=380)$} \\
\hline Manhã & 81 & 21,3 \\
\hline Tarde & 212 & 55,8 \\
\hline Noite & 87 & 22,9 \\
\hline \multicolumn{3}{|l|}{ Motivo da escolha do periodo de procura $(n=380)$} \\
\hline Indisponibilidade nos demais períodos & 75 & 19,7 \\
\hline Acredita ser um período de atendimento mais rápido & 64 & 16,8 \\
\hline Menor número de pessoas & 62 & 16,3 \\
\hline Trabalha no período da manhã & 54 & 14,2 \\
\hline Período que inicio os sintomas & 52 & 13,7 \\
\hline Encaminhado pela UBS & 43 & 11,3 \\
\hline Horário que chegou do trabalho & 23 & 6,1 \\
\hline Solicitação da escola/creche do filho & 7 & 1,8 \\
\hline \multicolumn{3}{|l|}{ Primeira procura $(n=380)$} \\
\hline Sim & 47 & 12,4 \\
\hline Não & 333 & 87,6 \\
\hline \multicolumn{3}{|l|}{ Encaminhamento $(n=380)$} \\
\hline Sim & 48 & 12,6 \\
\hline Não & 332 & 87,4 \\
\hline
\end{tabular}

Fonte: Do autor.

\section{Discussão}

Neste estudo pode-se observar uma grande procura pelo PA em detrimento da atenção primária, uma vez que apenas $22,7 \%$ procuraram primeiro o serviço da UBS.

Cabe salientar que a procura pelo PAI se deve a insatisfação com o atendimento prestado na UBS (49,5\%), sendo referido como principais causas a 
falta de profissionais, a demora no atendimento e a dificuldade para o agendamento de consultas.

As causas da procura pelo PA e a insatisfação com os serviços da atenção primária também foi evidenciado no estudo de Kovacs et al. (2005), referindo que a relação oferta e demanda na rede básica continua expressando o confronto de interesses e necessidades, o qual propicia a procura pelos serviços de maior densidade tecnológica para problemas de saúde que, olhados da perspectiva técnica, poderiam ser resolvidos no nível primário.

A implantação do Sistema Único de Saúde (SUS) ocorrida no início da década de 90 tinha como principal objetivo garantir a ampliação do acesso da população aos serviços de saúde. Mais recentemente, a edição do pacto pela saúde propôs novas diretrizes para as políticas de saúde, reafirmando a municipalização e a organização do sistema, por meio da atenção básica, como caminho para a consolidação do SUS (CUNHA; VIEIRA, 2010).

Dessa forma, a acessibilidade aos serviços de saúde representa um importante componente de um sistema de saúde no momento em que se efetiva o processo de busca e obtenção do cuidado (CUNHA; VIEIRA, 2010). Para Donabedian (1988), trata-se daquelas características dos serviços que permitem que os mesmos sejam mais facilmente utilizados pelos usuários. Desse modo, os resultados deste estudo evidenciam dificuldade de acessibilidade junto as UBS, o que se justifica pela grande procura pelo PA.

Este fato demonstra uma desigualdade entre a oferta e a demanda do serviço, gerando uma inacessibilidade, causado pelas filas de espera, falta de oferta do serviço procurado, assim como uma descontinuidade do atendimento em saúde. A falta do cumprimento do princípio de integralidade coloca em risco a saúde do cidadão, que desmotivado pela demora no atendimento, acaba desistindo ou procurando outros serviços (MILBRATH et al., 2009).
Vale ressaltar que dos $26,2 \%$ que procuraram por outro atendimento antes do PAI, apenas $22,7 \%$ de toda a amostra tiveram a UBS como porta de entrada, sendo que a maioria procurou primeiro pelo PAI, sendo o período vespertino, o horário mais procurado; no entanto, sabe-se que as UBS estão em funcionamento. Evidenciando que a população tem utilizado o PA como porta de entrada do serviço de saúde.

As unidades de PA servem para o atendimento de urgências de menor complexidade, pois são unidades de atendimento intermediário entre as unidades básicas de saúde e as portas de urgência hospitalares. O PA proporciona assistência médica descontínua, focado na queixa, ficando a cargo das unidades básicas uma assistência contínua e integral através da orientação, prevenção e quando necessário o encaminhamento.

Este estudo evidenciou que a grande maioria procurou pelo PA sem possuir encaminhamento, o que nos remete a refletir sobre o conceito da integralidade que exige a identificação adequada das necessidades de saúde dos usuários, fornecendo recursos para manejo e resolutividade, como aqueles identificados no encaminhamento de crianças para atenção especializada. Trata-se de um mecanismo importante que garante o ajustamento de necessidades de serviços de saúde. Assim, os profissionais de saúde têm a responsabilidade de identificar as necessidades de saúde e fornecer e coordenar os serviços nas unidades de atenção primária, encaminhando apenas os casos que não podem ser tratados nessas unidades (FURTADO et al., 2013). Para tanto, é necessário que os pais/ responsáveis possam ter acesso imediato à atenção primária.

Todavia, observa-se que os pais/responsáveis, em sua maioria, preferem o PA por relacionálo a uma imagem de melhor acessibilidade e resolubilidade. Acreditam que o atendimento na UBS seja restrito e não atenda às suas necessidades, por vezes não compreendem e/ou desconhecem a 
função do PA ou realmente acreditam que seu caso é grave por desconhecer o conceito de urgência. Consequentemente, gera aumento dos custos e necessidade de recursos humanos, dificultando assim a assistência de verdadeiras urgências (SANTOS et al., 2013).

O acolhimento, proposta de reorganização dos serviços para garantir acesso, resolubilidade e atendimento humanizado, é uma tentativa de minimizar esses obstáculos. Contudo, além das dificuldades da rede de serviços, a demanda espontânea traduz outras formas de conceber, valorizar e agir frente ao adoecimento (KOVACS et al., 2005).

Este conceito fica evidente ao observamos os resultados deste estudo no que tange as causas da procura, sendo a febre $(25,7 \%)$ a principal, o que confirma os dados encontrados no estudo de Feldhaus e Cancelier (2012), no qual a febre é uma das queixas mais frequentes em consultas pediátricas, não somente ao nível ambulatorial, como também em emergências.

Já as queixas classificadas como verdadeiras urgências, como acidentes (2,9\%), sangue nas fezes $(0,7 \%)$ e convulsão $(0,4 \%)$ teve uma baixa prevalência, o que nos remete a pensar que os cuidadores utilizam o serviço de urgência para causas clínicas.

Estes dados vêm corroborar com o pensamento de Oliveira et al. (2012), que refere que o funcionamento das unidades de saúde, baseadas no agendamento de consultas para atender casos clínicos de doenças agudas, por demanda espontânea, não tem resolvido os problemas de saúde, por não dar conta de atender a todos que procuram o serviço ou por carecer de resolutividade. Ademais, a atenção programada não é executada como prática das unidades. Apontando também a (des)humanização do cuidado nesse sistema, em que pessoas precisam estar de madrugada à porta das unidades de saúde para conseguir o atendimento que procuram por demanda espontânea.

\section{Conclusão}

$\mathrm{O}$ presente estudo revelou grande procura da população estudada pelos serviços do PA em detrimento da atenção primária, o que confirma a deficiência em alguns princípios prioritários do SUS, como acessibilidade e resolutividade dos serviços.

Este estudo também permitiu identificar que as causas de procura dos pais/responsáveis pelo serviço do PA poderiam ter sido resolvidas na atenção primária. Portanto, há necessidade de reestruturação na rede de saúde do município buscando principalmente a integralidade, acessibilidade e resolutividade da assistência à criança.

Desse modo, seria possível um atendimento de qualidade na atenção primária e não ocorreria o deslocamento da criança para o PA e, consequentemente, haveria prioridade e agilidade dos casos graves e doenças agudas.

Considerando estes pontos, há necessidade também de uma reeducação da população sobre o conceito de atenção básica e atendimento emergencial.

\section{Referências}

BRASIL. Ministério da Saúde. Conselho Nacional de Saúde. Resolução no 466 de 12 de dezembro de 2012: aprova as diretrizes e normas regulamentadoras de pesquisas envolvendo seres humanos. Brasília, DF, 2012. Disponível em: <http://www.uel.br/comites/ cepesh/pages/arquivos/Resolucao\%20CNS\%204662012.pdf>. Acesso em: 25 abr. 2014.

- Manual Instrutivo da rede de atenção às urgências e emergências no Sistema Único de Saúde (SUS). Brasília, 2013. Disponível em: <http://bvsms. saude.gov.br/bvs/publicacoes/ manual_instrutivo_ rede_atencao_urgencias.pdf $>$ Acesso em: $10 \mathrm{abr}$. 2014 .

. Secretaria de Atenção à Saúde. Departamento de Atenção Básica. Política Nacional de Atenção. 4. ed. Brasília, 2007. Disponível em: <http://portal. saude.gov.br/portal/arquivos/ pdf/volume_4 completo.pdf $>$. Acesso em: 25 abr. 2014. 
CUNHA, A. B. O.; VIEIRA, L. M. S. Acessibilidade aos serviços de saúde em um município do Estado da Bahia, Brasil, em gestão plena do sistema. Caderno de Saúde Pública, Rio de Janeiro, v. 26, n. 4, p.725-737, abr. 2010.

DONABEDIAN, A. A. Los espacios de la salud: aspectos fundamentales de la organización de la atención médica. México, DF: Biblioteca de la Salud; 1988.

FELDHAUS, T.; CANCELIER, A. C. L. Conhecimentos dos pais sobre febre em crianças. Arquivos Catarinenses de Medicina, Florianópolis, v. 41, n. 1, p. 16-21. 2012.

FURTADO, M. C. C.; BRAZ, J. C.; PINA, J. C.; MELLO, D. F.; LIMA, R. A. G. A avaliação da atenção à saúde de crianças com menos de um ano de idade na Atenção Primária. Revista Latino-Americana de Enfermagem, Ribeirão Preto, v. 21, n. 2, mar./abr. 2013.

KOVACS, M. H.; FELICIANO, K. V. O.; SARINHO, S. W.; VERAS, A. A. C. A. Acessibilidade às ações básicas entre crianças atendidas em serviços de pronto-socorro. Jornal de Pediatria, Rio de Janeiro, v.81, n.3, p. 251-258, 2005.

MARQUES, G. Q.; LIMA, M.A. D. S. Demandas de usuários a um serviço de pronto atendimento e seu acolhimento ao sistema de saúde. Revista Latino-Americana de Enfermagem, Ribeirão Preto, v.15, p.13-19, jan./fev. 2007. Disponível em: <http://www.scielo.br/pdf/rlae/v15n1/pt v15n1a03.pdf $>$. Acesso em: 13 abr. 2014.

MILBRATH, V. M., AMESTOY, S. C.; SOARES, D. C.; SIQUEIRA, H. C. H. Integralidade e acessibilidade no cuidado à criança portadora de paralisia cerebral. Acta Paulista de Enfermagem, São Paulo, v.22, n. 6, p. 755-760, 2009.

OLIVEIRA, B. R. G.; VIERA, C. S.; COLLET, N.; LIMA, R. A. G. Acesso de primeiro contato na atenção primária em saúde para crianças. Revista da Rede de Enfermagem do Nordeste, Fortaleza, v.13, n.2, p. 332-342, 2012.
PEIXOTO, B. V.; PIAZZETTA, E.; RISCHINI, F. A.; GUIMARAES, M. N. C.; CUZIOL, M.; LODO, P. B.; BAUMGRATZ, T. D.; ZEPPONE, S. C. A difícil realidade do pronto atendimento infantojuvenil mostrando a situação de saúde de uma cidade. Revista Paulista de Pediatria, São Paulo, v.31, n. 2, p. 231-236, 2013.

SANTOS, M. L.; SILVA, R. B.; VOGT, M. S. L.; HAEFFNER, S. B.; MICHELOTTI, M. R.C. Pronto atendimento infantil: quem utiliza e por que motivo. Revista Saúde Santa Maria, Santa Maria, v. 39, n. 2 , p. 79-88, 2013.

SILVA, E. M. R.; TRONCHIN, D. M. R. Acolhimento de usuários em Pronto-Socorro Infantil na perspectiva dos enfermeiros. Acta Paulista de Enfermagem, São Paulo, v. 24, n. 6, p. 799-803, 2011.

STARFIELD, B. Atenção primária: equilíbrio entre necessidades de saúde, serviços e tecnologia. Brasília: UNESCO, Ministério da Saúde, 2002.
Recebido em: 25 mai. 2014. Aceito em: 24 nov. 2014. 
